Original article

\title{
Serotonin transporter gene polymorphism as a predictor of short-term risk of suicide reattempts
}

\author{
Federico M. Daray ${ }^{\mathrm{a}, \mathrm{b}, \mathrm{c}, *}$, Ángeles R. Arena ${ }^{\mathrm{a}}$, Arnaldo R. Armesto $^{\mathrm{a}}$, Demián E. Rodante ${ }^{\mathrm{a}, \mathrm{c}}$, \\ Soledad Puppo ${ }^{\mathrm{d}}$, Patricia Vidjen ${ }^{\mathrm{e}}$, Alicia Portela ${ }^{\mathrm{e}}$, Leandro N. Grendas ${ }^{\mathrm{a}}$, \\ Andrea E. Errasti ${ }^{\mathrm{a}} \mathrm{b}$ \\ a University of Buenos Aires, School of Medicine, Institute of Pharmacology, Argentina \\ ${ }^{\mathrm{b}}$ National Scientific and Technical Research Council (CONICET), Argentina \\ ${ }^{\mathrm{c}}$ Braulio A. Moyano Neuropsychiatric Hospital, City of Buenos Aires, Argentina \\ d Hospital de Clínicas José de San Martín, City of Buenos Aires, Argentina \\ e José Tiburcio Borda Hospital, City of Buenos Aires, Argentina
}

\section{A R T I C L E I N F O}

\section{Article history:}

Received 19 March 2018

Received in revised form 8 June 2018

Accepted 4 July 2018

Available online 20 July 2018

\section{Keywords:}

Suicide behavior

Impulsivity

5-HTTLPR

Polymorphism

Predictor

Serotonin

\begin{abstract}
A B S T R A C T
Objective: The serotonin-transporter-linked polymorphic region (5-HTTLPR) polymorphisms are associated with suicidal behavior; however, prospective studies are scarce. Herein we aim to determine if 5-HTTLPR polymorphisms predict risk of short-term suicide reattempt in a high-risk suicidal sample. We also explore possible mediators or moderators of this relationship.

Methods: A multicenter prospective cohort study was designed to compare data obtained form 136 patients admitted to the emergency department for current suicidal ideation or a recent suicide attempt. Subjects were clinically evaluated, genotyped, and monitored for a new suicide attempt for 6 months. Results: At 6 months of follow up, 21\% of the subjects had a new suicide attempt. The frequency of L-allele and L-carrier was higher in reattempters when compared with non-reattempters ( $55.8 \%$ vs. $35.4 \%, p=$ 0.01 and $76.9 \%$ vs. $54.2 \%, p=0.04$, respectively). Reattempters also differ from non-reattempters patients with respect to age, history of previous suicide attempts, and age of onset of suicidal behavior. The logistic regression model showed that L-carriers had an odds ratio of 2.8 (95\% CI: $1.0-7.6)$ for reattempts when compared to SS genotype. The adjusted model indicates that this association is not mediated or moderated by impulsivity.

Conclusion: The 5-HTTLPR polymorphisms predicted short-term risk of suicidal reattempt independently of age and sex. L-carriers have almost three times more risk of relapse when compared with SS carriers.
\end{abstract} (C) 2018 Elsevier Masson SAS. All rights reserved.

\section{Introduction}

Suicide and suicidal behavior (SB) have been classified as among the leading causes of death and injuries worldwide. Approximately 800,000 deaths by suicide occur annually with $10-$ 20 times more individuals attempting suicide, indicating that both suicide and non-fatal SB are prevalent problems that need to be addressed [1]. More than two thirds of patients completing suicide did so on the first attempt [2]. As such, interventions should focus on early detection and prevention of suicide attempts (SA). The study of subjects with SB is highly relevant to suicide mortality

\footnotetext{
* Corresponding author at: Instituto de Farmacología, Facultad de Medicina, Universidad de Buenos Aires, Paraguay 2155, piso 9, C1121ABG, Ciudad de Buenos Aires, Argentina.

E-mail address: fdaray@hotmail.com (F.M. Daray).
}

since a history of SA confers a 42-fold increased risk for suicide [3]. A limitation in assessing suicide risk among patients is that clinicians must rely on information provided by patients, and for different reasons, patients oftentimes may not provide accurate information about their suicidal status. Therefore, a need exists for the development of genomic, biochemical, molecular, imaging, and neuropsychological predictors for suicide risk.

Efforts to understand and predict SB must start with the study of potential contributing factors. Based on the stress-diathesis model, SB can be understood as a result of an interaction between state-dependent (environmental) stressors and trait-like diathesis $[4,5]$. The term diathesis or susceptibility is thought to include heritable factors among others, which could increase risk of SB. Twin and adoption studies have demonstrated that suicide has a heritable genetic component [6]. It has been estimated that the heritability for suicide ranges between $21-50 \%$ and between $30-$ $55 \%$ for a broader phenotype of suicidal behavior and ideation [7]. 
Offspring of probands who have attempted suicide are also at a nearly 5-fold higher risk of attempting suicide themselves. Although other psychiatric conditions associated with SB are also heritable, severe SB appears to be transmitted independently [8,9]. Since suicide and SB have a hereditary component, the first genetic studies used candidate gene association approaches to identify one or several genes variants that may increase the risk of SB, with most studies focused on biological systems linked to SB. Over the past decade, the field shifted to genome-wide association studies (GWAS), which use a less biased approach based on gene discovery. However, despite a great deal of enthusiasm and the potential to uncover novel genetic contributors to SB, GWAS studies collectively showed a lack of significant and reproducible findings [10], implying that individual gene variants are likely to account for only a small proportion of the total phenotypic variability [11]. Recent studies provided evidence that epigenetic mechanisms such as hypermethylation of brain derived neurotrophic factor (BDNF) could explain the missing link between heritability of SB and interaction with the environment [12].

Candidate genes studies for SB have been selected based on established biological correlates since alterations in serotonergic transmission have been observed in patients with SB [13-15] Genes involved in the synthesis (tryptophan hydroxylase, TPH), transport (serotonin transporter, 5-HTT), transmission (serotonin $1 \mathrm{~A}$ receptor, 5- $\mathrm{HT}_{1 \mathrm{~A}}$; serotonin $2 \mathrm{C}$ receptor, $5-\mathrm{HT}_{2 \mathrm{C}}$ ) and degradation (monoamine oxidase A; MAO-A) of serotonin have been used in association studies [16].

SLC6A4, which is located on chromosome 17q11.1-q12 and encodes for the serotonin transporter (5-HTT), is the most studied candidate gene and is responsible for regulating the duration of the serotonergic signal in the central nervous system (CNS) [17]. Several polymorphisms have been described for SLC6A4, but most studies have focused on a common polymorphism in the 5 promoter region, referred to as the serotonin-transporter-linked polymorphic region (5-HTTLPR) [18]. Even though 3 polymorphisms have been reported [19], most research has focused on two variations in this region that generate a short $(\mathrm{S})$ allele with 44 fewer base pairs than the long (L) allele. While in vitro studies provide evidence that variations in this region are associated with different basal activity of the transporter, which is most likely related to varying transcriptional activity [20], this has not been confirmed in in vivo studies [21].

Although the 5-HTTLPR is the most studied, studies employing this genetic marker use a cross-sectional design used to detect associations among these polymorphisms and SB in patients with different psychiatric diagnoses and comorbidities or between patients and control subjects [16]. Previous studies have shown contradictory results, with some suggesting an association between SB and L-allele or L-homozygotes while others report an association between SB and the S-allele or S-carriers [16].

Because one of the best predictors of a future SA and suicide is a previous SA [2], clinicians require tools to categorize high-risk suicidal subjects according to their potential risk and try to predict who may be more prone to relapse. To date, we lack robust genetic predictors that can help quantify suicide risk, and the only method to assess risk is using longitudinal cohort studies. To the best of our knowledge, only one prospective cohort study has assessed the role of 5-HTTLPR polymorphisms as a predictor of suicide events in high-risk suicidal patients in which SS genotype was associated with a higher risk of reattempts [22]. However, these results have not been replicated.

Impulsivity and aggression are two personality traits frequently associated with SB and meet the definition of endophenotype [23]. In addition, both impulsivity and aggressive behavior have been related to serotonergic abnormalities [24]. It has been proposed that these abnormalities in serotonergic function can influence neurobiological systems and cognitive functioning, resulting in personality developments, such as impulsivity and/or aggressive traits, that lead to SB, especially under the influence of acute stressors or psychopathological states [24]. Despite some inconsistencies, association studies have linked impulsive and aggressive behavior with 5-HTTLPR polymorphisms. An association between the $\mathrm{S}$-allele and increased aggressiveness and impulsivity has been described in various cohorts including children $[25,26]$, adolescents [27], adopted children [28], adolescent and young girls [29], cocaine-dependent individuals [30], and patients with personality disorders [31].

In the present study, we aimed to determine if the genetic status of 5-HTTLPR polymorphisms predicts the risk of short-term suicide reattempt in a high-risk suicidal patient sample. We also explored whether suicidal endophenotypes are mediators or moderators of this relationship. Taking into account prior studies, it was hypothesized that S-carrier status would predict short-term suicide reattempt.

\section{Methods}

\subsection{Study design}

The present study used blood samples obtained from patients enrolled in a multicenter prospective cohort study conducted in Buenos Aires, Argentina. The cohort was recruited from three different hospitals: the Braulio A. Moyano Neuropsychiatric Hospital, the Hospital Borda, and the Hospital de Clínicas "José de San Martin", in the city of Buenos Aires. All hospitals in the current study serve a large urban catchment area in Buenos Aires and predominantly treat low-income, uninsured patients. The cohort study began in 2012 with collection of baseline data finishing in December 2016. The current study utilized data obtained at 6 months of follow-up. The study protocol was approved by the institutional review board at each participating hospital.

\subsection{Patients}

Participants were patients who had been admitted to the emergency department of one of the three hospitals for current suicidal ideation (SI) or a recent SA. SI was defined as any current self-reported thought of engaging in suicide-related behavior [32], and SA was defined as a potentially self-injurious behavior with a nonfatal outcome, for which there was evidence (either explicit or implicit) that the person intended at some (non-zero) level to kill him or herself [33].

Eligible participants were aged 18-65 years, hospitalized for SI or a SA within $72 \mathrm{~h}$, sufficiently alert and able to respond with fluency in Spanish, and could provide written informed consent to participate. Participants were excluded if they were unable to respond autonomously (ie, due to sedative effects of medication or language limitations), were transferred to another institution, or had a profession related to mental health.

All participants gave written informed consent to participate in the study. Participants were included in the study if all relevant measures were completed at the baseline assessment. After discharge, subjects who had been recruited as inpatients received treatment as usual in the community. Participants were evaluated 6 months after their hospitalization.

\subsection{Measures}

\subsubsection{Baseline data}

At baseline evaluation, each participant underwent a semistructured interview conducted by one of three psychiatrists (LG, 
AP, and SP) in the emergency departments. All psychiatrists on the research team had at least 5 years of clinical experience and underwent training for the semi-structured interviews and datagathering procedures of the study. The semi-structured interview included questions specific to clinical and demographic variables. The Mini International Neuropsychiatric Interview (MINI) [34] and the Structured Clinical Interview for DSM-IV Axis II Disorders (SCID-II) [35] were used for diagnostic purposes. Participant history of sexual abuse, history of SA, age of first SA, hospitalizations due to SA, and family history of suicidal behavior and psychiatric illness were assessed during this interview. The Columbia-Suicide Severity Rating Scale (C-SSRS) [36] was used to obtain further details related to a participant's lifetime and most recent SB. To assess impulsivity, participants were administered the Barratt Impulsiveness Scale (BIS)-11 [37]. The BIS-11 is composed of 30 items describing common impulsive or nonimpulsive (for reverse scored items) behaviors and preferences. The BIS-11 yields three second-order factor scores: attentional impulsiveness (ie, intrusive thoughts and racing thoughts), motor impulsiveness (ie, tendency to act on the spur of the moment and consistency of lifestyle), and nonplanning impulsiveness (ie, difficulties with careful thinking and planning or enjoyment of challenging mental tasks). The Buss-Durkee Hostility Scale (BDHS) was used to evaluate the different aspects of hostility [38]. It consists of 75 items that are grouped into seven subscales of hostility and one subscale of guilt. The items are of "true and false" dichotomous response. It provides a total score and scores on each of the eight subscales; from the clinical point of view, the total score is more relevant. The Beck Hopelessness Scale (BHS) was used to assess aspects of hopelessness [39]: the negative expectations that a person has about his future and well-being as well as his ability to overcome difficulties and achieve success in his life. It is a 20-item self-report inventory to which an individual answers "true or false." Recent stressors were assessed with the Brugha Stressful Life Events Scale (List of Threatening Experiences [LTE]) [40]. The LTE is an instrument designed to collect the existence of stressful life events that occurred during a specific time period (recommended to be 6 months prior). It is a self-applied instrument that consists of a list of 12 categories of stressful life events to which individuals must answer "Yes" ( 1 point) or "No" ( 0 points). A global score is obtained that consists of the sum of the scores obtained in each of the 12 items.

At the end of the baseline assessment, blood samples were collected into EDTA blood collection tubes (BD Vacutainer) and transported to the laboratory in the Pharmacology Institute of the School of Medicine of the University of Buenos Aires, Argentina.

\subsubsection{Follow-ups}

Two trained psychiatrists (DR and LG) performed the telephone follow-ups. Participants were contacted by telephone at 6 months following their baseline assessment. If participants could not be reached, calls were made on alternate hours and days for one week. If contact was still not achieved, interviewers contacted two reference numbers that was provided upon enrollment. If contact was still not possible, an e-mail was sent. If contact was not established after three attempts, the participant was declared as "loss to follow-up."

When interviewers contacted the participants or the reference person, they assessed whether participants experienced a new SA during the follow-up period.

\subsection{Genetic analysis}

Genomic DNA was obtained from EDTA anti-coagulated peripheral blood using the ReliaPrep Blood gDNA (Promega) and DNeasy Blood and Tissue Kit (Quiagen) columns according to manufacturer specifications. Polymerase chain reaction (PCR) amplification of 5-HTTLPR polymorphism was carried out in a total volume of $50 \mu \mathrm{l}$ containing $10 \mu \mathrm{l}$ buffer, $1.5 \mathrm{mM} \mathrm{MgCl}, 10 \mu \mathrm{l}$ 5X-PCR enhancer (PCR MAX, Biodynamics), $5 \mathrm{pmol}$ of each primer (Sigma Aldrich), $0.3 \mathrm{mM}$ dNTP mix, 0.5 units of GoTaq Hot Start DNA Polymerase (Promega) and 50-100 ng DNA sample. We amplified the 5-HTT regulatory region using the following primers: 5'-GAGGGACTGAGCTGGACAACCAC-3' (reverse) and 5'GCGTTGCCGCTCTGAATGC-3' (forward). The PCR was performed as follows: an initial denaturation step of $94^{\circ} \mathrm{C}$ for $3 \mathrm{~min}$, followed by 38 cycles of $94^{\circ} \mathrm{C}$ for $1 \mathrm{~min}, 55^{\circ} \mathrm{C}$ for $1 \mathrm{~min}$, and $72^{\circ} \mathrm{C}$ for $1 \mathrm{~min}$, and a final extension step at $72^{\circ} \mathrm{C}$ for $7 \mathrm{~min}$. PCR products were separated on $2 \%$ agarose gel stained with Gel Red (Biotium) to allow identification of the Lallele (572 bp) and the S allele (528 bp). Genotyping procedures were conducted by researchers (ARA and AEE) who were blinded to the clinical status of the patients.

\subsection{Data analysis}

Categorical measures are reported as frequencies or percentages, and continuous measures are reported as means \pm standard deviations (SD). Comparisons between groups depend on the type of variable: categorical measures were compared with contingency tables $\left(x^{2}\right)$ and Fisher's exact test, continuous measures by ANOVA methods (t-test), or Wilcoxon rank-sum test (Mann-Whitney Ustatistic) for non-normally distributed continuous data.

Odds ratios (OR) and 95\% confidence intervals $(\mathrm{CI})$ were used to evaluate the association between suicide reattempt and genetic status via multivariable-adjusted logistic regression models. Independent variables were included stepwise and alpha $(\alpha)$ to enter was set at 0.20 . Beta $(\beta)$ coefficients and their 95\% CI from logistic regression modeling are reported, with significance evaluated using t-test. Statistical significance was set at two-tailed $P<0.05$. Assumptions were tested, and a robust analysis was performed to adjust our model to account for heteroscedasticity. All statistical analyses were conducted using SPSS software version 25 .

\section{Results}

One hundred thirty-six participants (aged $37.1 \pm 13.1$ years with $66.2 \%$ women) fulfilled all the specified inclusion criteria and consented to the study. Of the total population, 85 (64\%) participants met DSM-IV diagnostic criteria for current major depressive disorder (MDD), 25 (19\%) for schizophrenia, and 20 (15\%) for bipolar disorder (BD). A comorbid borderline personality disorder (BPD) was reported in $46 \%$ of the population, and substance abuse in $21 \%$. Allele frequencies were 163 (59.9\%) for the S-allele and 109 (40.1\%) for the L-allele. Distribution of the genotypes were 57 (42\%) for SS homozygotes, 49 (36\%) for SL heterozygotes, and 30 (22\%) for LL homozygotes. The distribution of genotype frequencies of 5-HTTLPR did not show any divergence from the Hardy-Weinberg equilibrium $\left(x^{2}=5.03, P=0.08\right)$.

At baseline, we found no significant differences in genotype status among patients with SI (SS-genotype, 41.3\%, SL-genotype, $39.1 \%$, LL-genotype, 19.6\%), single suicide attempters (SS-genotype, $58.8 \%$, SL-genotype, 23.5\%, LL-genotype, 17.6\%), and multiple suicide attempters (SS-genotype, 32.1\%, SL-genotype, 41.1\%, LLgenotype, $26.8 \%)(P=0.16)$. When comparing baseline allele status among groups with SI (S-allele, 60.9\%, L-Allele, 39.1\%), single suicide attempters (S-allele, 70.6\%, L-Allele, 29.4\%), and multiple suicide attempters (S-allele, 52.7\%, L-Allele, $47.3 \%$ ) we observed a statistical tendency $(P=0.06)$ towards a higher frequency of the Lallele in the group of subjects with multiple suicide attempts.

Participants who were lost during follow-up differed from those available for the analysis, specifically in the diagnosis of bipolar disorder $(P=0.03)$ and family history of suicide attempts 
$(P=0.02)$. Other clinical and demographic variables are shown in Table 1.

At 6 months of follow up, 26 (21\%) subjects reported a new suicide attempt. Reattempters differ from non-reattempters with respect to age (33.0, $95 \% \mathrm{CI}: 28.5$ to 37.5 vs $38.5,95 \% \mathrm{CI}: 35.8$ to $42.3, P=0.05)$, history of previous suicide attempts $(84.6 \%$ vs $62.5 \%, P=0.04)$, and age of first suicide attempt (23.4, 95\% CI: 19.0 to 27.8 vs $29.8,95 \% \mathrm{CI}: 26.8$ to $32.9, P=0.05$ ). Furthermore, the frequency of L-allele was higher in reattempters when compared with non-reattempters (55.8\% vs $35.4 \%, P=0.01$ ). L-carriers' genotypes were different in reattempters when compared with non-reattempters $(76.9 \%$ vs. $54.2 \%, P=0.04)$. Of the different psychopathological traits evaluated, only impulsivity was higher in subjects with suicide reattempts and trended towards statistical significance $(69.3,95 \% \mathrm{CI}$ : 66.4 to 72.2 vs $73.6,95 \% \mathrm{CI}: 67.4$ to 79.84 , $P=0.19)$. There were no significant differences in other clinical or demographic variables between both groups as shown in Table 2 .

Unadjusted and adjusted ORs for the association of suicide reattempt with genetic status are presented in Table 3. In unadjusted models, the OR for suicide reattempt was almost 3 times higher in L-carrier (those with L allele in their genotype: LL or LS) when compared to SS genotype (OR $=2.8,95 \% \mathrm{CI}: 1.0-7.6)$. A multivariable logistic regression modeling was then performed to evaluate the role of impulsivity on the relation between genetic status and suicide reattempt. After adjusting for impulsivity, age, and sex, the OR for LL genotype was not significantly different $(\mathrm{OR}=3.3 ; 95 \% \mathrm{CI}: 1.1-9.8)$. Moreover, the interaction term between L-carrier and impulsivity was not significant $(p=0.18)$.

\section{Discussion}

The present findings indicate that the L-carrier genotypes of the 5-HTTLPR polymorphism predict short-term suicide reattempt in high-risk suicidal patients. This relation was not mediated or moderated by impulsivity. In addition, subjects who reattempted suicide at 6 months of follow-up were more likely to be younger, have a history of previous SA, and experienced their first SA at younger age.

Epidemiological studies suggest that suicide and SB is, at least partially, genetically determined with a pattern of transmission independent of the genetic transmission of psychiatric disorders [41]. Although the pathological mechanisms that lead to SB have not yet been elucidated, one of the most consistent findings is the observation of a reduced serotonergic function [13,42]. Abnormalities in serotonergic function have been observed in postmortem autoradiography studies showing a decrease in 5-HTT binding and an increases in $5-\mathrm{HT}_{1 \mathrm{~A}}$ receptor binding together with a reduction in the 5-HIAA metabolite, suggesting reduction in serotonergic function, specifically in the anterior cingulate and ventral prefrontal cortex (PFC) regions involved in behavioral inhibition and decision making [14]. This observation has been supported by recently published in vivo studies using positron emission tomographic (PET) imaging, showing that individuals with MDD who attempt suicide have lower midbrain serotonin transporter binding compared with those who do not attempt suicide and that higher 5HT1 A binding in raphe nuclei is associated with higher lethality of SB [43]. Moreover, longitudinal prospective studies using PET shown that greater $5-\mathrm{HT}_{1 \mathrm{~A}}$ binding predict higher SI and more lethal suicidal behavior in a 2-year follow-up period [44]. Altogether, these findings confirm the initial observations associating reduced serotonergic function with SB. For this reason, many studies with candidate genes are focused on the associations among genes, the serotonergic system, and SB. Among those genes, the one encoding for the 5-HTT has captured the most attention since it regulates the duration and the magnitude of the serotonergic signal [45]. The

Table 1

General characteristics of the suicidal patients at index admission.

\begin{tabular}{|c|c|c|c|c|}
\hline & $\begin{array}{l}\text { (1) All patients included in the } \\
\text { study }\end{array}$ & $\begin{array}{l}\text { (2) Patients that complete the follow- } \\
\text { up }\end{array}$ & $\begin{array}{l}\text { (3) Patients lost during the follow- } \\
\text { up }\end{array}$ & $\begin{array}{l}\text { P-Value (test) } \\
\text { (2) vs. (3) }\end{array}$ \\
\hline No. of participants & 136 & 122 & 14 & \\
\hline Age (yrs.), mean (CI 95\%) & $37.1(34.8-39.3)$ & $37.3(35.0-39.8)$ & $34.6(27.1-42.1)$ & 0.46 \\
\hline Women $(\%)$ & $90(66.2 \%)$ & $81(66.4 \%)$ & $9(64.3 \%)$ & 1.00 \\
\hline \multicolumn{5}{|l|}{ Diagnosis (\%) } \\
\hline Major depression & $85(64.4 \%)$ & $79(66.4 \%)$ & $6(46.1 \%)$ & 0.22 \\
\hline Bipolar Disorder & $20(15.1 \%)$ & $15(12.6 \%)$ & $5(38.5 \%)$ & 0.03 \\
\hline Schizophrenia & $25(18.9 \%)$ & $23(19.3 \%)$ & $2(15.4 \%)$ & 1.00 \\
\hline Other & $2(1.5 \%)$ & $2(1.7 \%)$ & $0(0.0 \%)$ & 1.00 \\
\hline \multicolumn{5}{|l|}{ Co-occurring disorders (\%) } \\
\hline Borderline personality & $62(46.3 \%)$ & $56(46.7 \%)$ & $6(42.8 \%)$ & 1.00 \\
\hline Substance abuse & $28(21.2 \%)$ & $23(19.2 \%)$ & $5(41.7 \%)$ & 0.13 \\
\hline \multicolumn{5}{|l|}{ Family history (\%) } \\
\hline Any psychiatric illness & $107(78.7 \%)$ & $96(78.7 \%)$ & $11(78.6 \%)$ & 1.00 \\
\hline Suicide or suicide attempt & $61(44.8 \%)$ & $59(48.4 \%)$ & $2(14.3 \%)$ & 0.02 \\
\hline \multicolumn{5}{|l|}{ Suicide history } \\
\hline Attempted previously (\%) & $90(66.2 \%)$ & $82(67.2 \%)$ & $8(57.1 \%)$ & 0.55 \\
\hline $\begin{array}{l}\text { Age at first attempt (yrs.), mean (CI } \\
95 \%)\end{array}$ & $28.2(25.8-30.6)$ & $28.4(25.8-31.0)$ & $26.2(19.9-32.4)$ & 0.59 \\
\hline \multicolumn{5}{|l|}{ Rating scale scores, mean (CI 95\%) } \\
\hline Impulsivity (BIS) & $70.4(68.0-72.8)$ & $70.2(67.6-72.8)$ & $72.6(66.5-78.8)$ & 0.53 \\
\hline Hopelessness (BHS) & $10.0(9.2-10.8)$ & $9.9(9.1-10.7)$ & $10.4(6.9-13.9)$ & 0.82 \\
\hline Hostility (BDHI) & $42.9(41.1-44.6)$ & $42.6(40.8-44.5)$ & $44.7(39.0-50.4)$ & 0.48 \\
\hline Recent stressors (SLE) & $311.3(283.9-338.7)$ & $306.1(277.4-334.8)$ & $355.4(256.2-454.6)$ & 0.28 \\
\hline History of Sexual abuse & $64(47.4 \%)$ & $56(46.3 \%)$ & $8(57.1 \%)$ & 0.57 \\
\hline \multicolumn{5}{|l|}{ Allele Status } \\
\hline S-Allele & $163(59.9 \%)$ & $147(60.2 \%)$ & $16(57.1 \%)$ & 0.84 \\
\hline L-Allele & $109(40.1 \%)$ & $97(39.8 \%)$ & $12(42.8 \%)$ & \\
\hline \multicolumn{5}{|l|}{ Genotype } \\
\hline SS genotype & $57(41.9 \%)$ & $50(41.0 \%)$ & 7 (50.0\%) & 0.16 \\
\hline SL genotype & 49 (36.0\%) & 47 (38.5\%) & $2(14.3 \%)$ & \\
\hline LL genotype & $30(22.0 \%)$ & 25 (20.5\%) & $5(35.7 \%)$ & \\
\hline
\end{tabular}

Rating scales: BIS = Barratt Impulsiveness Scale; BHS = Beck Hopelessness Scale; BDHI = Buss-Durkee Hostility Inventory; SLE = Stressful Life Events Scale. 
Table 2

Estimated prevalence and 95\% confidence intervals among reattempters and non-reattempters during the 6 month follow-up period.

\begin{tabular}{|c|c|c|c|}
\hline Variables & Non-reattempters & Reattempters & $P$-Value \\
\hline No. of study participants & 96 & 26 & \\
\hline Age (yrs.'), mean (CI 95\%) & $38.5(35.8-42.3)$ & $33.0(28.5-37.5)$ & 0.05 \\
\hline \multicolumn{4}{|l|}{$\operatorname{Sex}(\%)$} \\
\hline Women & $63(65.6 \%)$ & $18(69.2 \%)$ & \multirow[t]{2}{*}{0.82} \\
\hline Men & $33(34.4 \%)$ & $8(30.8 \%)$ & \\
\hline \multicolumn{4}{|l|}{ Diagnosis (\%) } \\
\hline Major depression & $62(66.7 \%)$ & $17(65.4 \%)$ & 1.00 \\
\hline Bipolar Disorder & $13(14.0 \%)$ & $2(7.7 \%)$ & 0.52 \\
\hline Schizophrenia & $16(17.2 \%)$ & $7(26.9 \%) 0$ & 0.27 \\
\hline Other & $2(2.1 \%)$ & $(0.0 \%)$ & 1.00 \\
\hline \multicolumn{4}{|l|}{ Co-occurring disorders (\%) } \\
\hline Borderline personality & $44(45.8 \%)$ & $12(50.0 \%)$ & 0.82 \\
\hline Substance abuse & $19(20.0 \%)$ & $4(16.0 \%)$ & 0.78 \\
\hline \multicolumn{4}{|l|}{ Family history $(\%)$} \\
\hline Any psychiatric illness & $76(79.2 \%)$ & $20(76.9 \%)$ & 0.79 \\
\hline Suicide or suicide attempt & $48(50.0 \%)$ & $11(42.3 \%)$ & 0.51 \\
\hline \multicolumn{4}{|l|}{ Suicide history } \\
\hline Attempted previously (\%) & $60(62.5 \%)$ & $22(84.6 \%)$ & 0.04 \\
\hline Age at first attempt (yrs) mean (CI 95\%) & $29.8(26.7-32.9)$ & $23.4(19.0-27.8)$ & 0.05 \\
\hline \multicolumn{4}{|l|}{ Rating scale scores mean (CI 95\%) } \\
\hline Impulsivity (BIS) & $69.3(66.4-72.2)$ & $73.6(67.4-79.8)$ & 0.19 \\
\hline Attentional & $19.4(18.5-20.4)$ & $20.9(18.8-23.0)$ & 0.19 \\
\hline Motor & $23.7(22.4-25.0)$ & $25.8(23.0-28.74)$ & 0.14 \\
\hline Nonplanning & $26.2(24.8-27.6)$ & $26.8(23.9-29.8)$ & 0.67 \\
\hline Hopelessness (BHS) & $9.8(9.0-10.7)$ & $10.2(7.9-12.5)$ & 0.73 \\
\hline Hostility (BDHI) & $42.0(40.0-44.1)$ & $44.8(40.3-49.2)$ & 0.25 \\
\hline Recent stressors (SLE) & $298.9(268.7-329.1)$ & $333.7(277.4-334.8)$ & 0.33 \\
\hline History of Sexual abuse & $44(46.3 \%)$ & $12(46.1 \%)$ & 1.00 \\
\hline \multicolumn{4}{|l|}{ Allele Status } \\
\hline S-Allele & $124(64.6 \%)$ & $23(44.2 \%)$ & 0.01 \\
\hline L-Allele & $68(35.4 \%)$ & $29(55.8 \%)$ & \\
\hline \multicolumn{4}{|l|}{ Genotype } \\
\hline SS genotype & 44 (45.8\%) & $6(23.1 \%)$ & 0.05 \\
\hline SL genotype & $36(37.5 \%)$ & $11(42.3 \%)$ & \\
\hline LL genotype & $16(16.7 \%)$ & $9(34.6 \%)$ & \\
\hline SS genotype & $44(45.8 \%)$ & $6(23.1 \%)$ & 0.04 \\
\hline L-carrier & $52(54.2 \%)$ & $20(76.9 \%)$ & \\
\hline
\end{tabular}

Rating scales: BIS = Barratt Impulsiveness Scale; BHS = Beck Hopelessness Scale; BDHI = Buss-Durkee Hostility Inventory; SLE = Stressful Life Events Scale.

gene that codes for the 5-HTT has a common functional promoter polymorphism (5-HTTLPR, rs4795541), which consists of a short (S) and a long (L) allele.

A cross-sectional design has been used in most studies aimed at exploring candidate genes to detect associations between 5-HTTLPR polymorphism and suicide or SA among patients with different psychiatric diagnoses or comorbidities or between patients and healthy controls [16]. Initial studies report an association between the L-allele of the 5-HTTLPR polymorphism and depressed patients who die by suicide when compared with control subjects [46]. However, subsequent studies failed to replicate these findings. For example, some studies found an association between the S-allele or S-carrier genotypes and SB [47-54], while other studies indicate no association at all [47,5558]. A possible explanation for the discrepancies among association studies could be that a single gene polymorphism may not explain complex multi-determined behaviors such as SB. Certain

Table 3

Unadjusted and adjusted odds ratios (ORs) and \% confidence intervals (95\% CIs) for the association of risk factors for reattempters.

\begin{tabular}{lll}
\hline Risk factors & Unadjusted OR $(95 \% \mathrm{CI})$ & Adjusted OR $(95 \% \mathrm{CI})^{1}$ \\
\hline Genetic Status (L-carrier) & $2.8(1.0-7.6)$ & $3.3(1.1-9.8)$ \\
Impulsivity (BIS score / 10) & $1.2(0.9-1.8)$ & $1.2(0.8-1.8)$ \\
Female & $1.2(0.5-3.0)$ & $1.1(0.4-3.1)$ \\
Age (decades) & $0.7(0.5-1.0)$ & $0.8(0.5-1.1)$ \\
\hline
\end{tabular}

1 Adjusted for all significant variables. L-carrier represents those patients with LL or LS genotypes. BIS = Barratt Impulsiveness Scale. gene expressions could be modified through epigenetic mechanisms such as DNA methylation or histone marks that modify expression levels and alter the response to stressors. Regulation of SLC6A4 gene expression may contribute to the inter-individual susceptibility or resilience [59]. Recent findings suggest a role of SLC6A4 AluJb methylation in MDD, amygdala reactivity, and stress reaction [60]; however, whether this mechanism is important in SB remains to be clarified. Future studies should not only address the presence or absence of one genetic polymorphism but also the possibility of epigenetic modifications.

The contradictory findings from candidate gene studies generated uncertainty about the usefulness of this polymorphism as a genetic marker of suicide or some type of specific SB (violent or lethal SB) because while 5-HTTLPR polymorphisms are some of the most studied, the underlying mechanism contributing to the variability in this relation is still unknown. However, some hypotheses have been proposed. In vitro studies with membrane preparations of lymphoblast suggested that the LL-genotype is associated with an increase in SLC6A4 transcription, resulting in increased transporter levels and more rapid serotonin uptake $[45,61]$. Taken together, it could be hypothesized that subjects with the L-allele or the LL-genotype have a reduction in extracellular serotonin and therefore, lower serotonin levels, which have been linked to SB as proposed by Du et al. 1999 [46]. A similar assumption has been used by those who found Scarriers to be associated with SB. In this case, the S-allele was associated with a decreased re-uptake of serotonin in individuals with SB [50]. Nonetheless, in vivo studies did not find the same 
impact of genetic polymorphism on serotonin neurotransmission as compared to in vitro studies [21]. Therefore, the "static hypothesis" on how 5-HTTLPR polymorphisms could be linked to SB by affecting serotonin bioavailability may not be useful. Another hypothesis that may explain a possible mechanism by which different genotypes may affect SB derives from the influence of the 5-HTTLPR allele status on brain development and neurogenesis. Evidence demonstrates that serotonergic neurons are among the first neurons generated and that serotonin plays an important role in brain development [62]. The importance of serotonin in neurogenesis has also been demonstrated [63], and this effect is potentially mediated through the interaction of the serotonergic system with BDNF. It has been demonstrated that during brain development, serotonin influences cortical development [64] and has neurotrophic effects on the hippocampus and frontal cortex [65]. The main finding of this study is that independent of age and sex, patients with L-allele in their genotype (L-carrier) are almost three times more at risk of a new suicide attempt after 6 months following hospitalization for SI or SA. Furthermore, we observed that participants who reattempt suicide during follow-up have a history of previous SA and start their SB at a younger age. Altogether, these observations are better explained by a "developmental hypothesis" in which sustained changes in the morphology and connections of certain brain regions could explain SB rather than a "statistic hypothesis" that explains circumstantial availability of serotonin. Alterations in gray matter brain volume [66] and connectivity [67] in the CNS have been associated with the Lallele of 5-HTTLPR polymorphism.

To our knowledge, the predictive value of 5-HTTLPR polymorphisms has only been studied in one genetic follow-up study with a cohort of high-risk subjects [22]. In that study, the S-carriers were characterized by a higher risk of repeated SB [22]. Taking into account prior published studies, it was hypothesized that the Scarriers status would predict short-term suicide reattempt. However, based on the results of this study, our initial research hypothesis was rejected. In our cohort, S-carrier status did not predict relapse but L-allele and L-carrier status did. This is consistent with the finding that at baseline multiple suicide attempters showed a higher frequency of L-allele when compared with participants with SI or single suicide attempt. A possible explanation for this discrepancy is that in the study of Courtet et al, only 76 patients ( $85 \%$ women) were included, and only 2 patients with the LL-genotype reattempted suicide. In this study, the total population included $60 \%$ more participants, and almost one-third identified as male.

5-HTTLPR polymorphisms have also been associated with endophenotypes, or categories of SB such as impulsive suicides [52]. In this study, we compared different characteristics of the SB of participants included in the sample population. Among all of these characteristics, impulsivity was the only one that exhibited a trend to be related to suicide reattempt; therefore, a logistic regression model was built to determine its role as mediator or moderator. Impulsivity, measured with the BIS-11, was neither a mediator nor a moderator of the relation between L-carrier genotypes and suicide reattempt.

Suicide behavior is determined by a combination of genetic and environmental interactions. In the last years, the 5-HTTLPR polymorphism was also found to increase the risk of developing SB following exposure to environmental factors such as child abuse [68] and stressful life events [69]. Therefore, in the present study, we explored if child sexual abuse or stressful life events in the year prior to hospitalization differed between those with or without reattempts at 6 month. Our results showed no differences between the groups.

The strength of this study relied on its prospective longitudinal design that allows exploring predictors of a SA. Furthermore, this study included several key factors that were measured using validated instruments, which might explain the relationship between the 5-HTTLPR polymorphism and SB. Despite these strengths, some limitations should be considered when interpreting the results because this study was limited to a unique patient sample hospitalized for severe SI or SA, which limits generalization of the results. Furthermore, we employed a biallelic approach; however, it is worth noting that there are additional polymorphic variants in the 5-HTTLPR. Recently, a single nucleotide polymorphism rs25531 $(A \rightarrow G)$ has been described for the L-allele, giving rise to variants of this allele denoted LA and LG [70]. The LG variant results in a reduction of the transcriptional efficiency of SLC6A4 in a similar manner to the S-allele. This could lead to a misclassification' however, the LG variant is absent or near absent in Caucasians and Hispanics population [71]. Moreover, an additional limitation was the limited assessment of early life stress; information was only gathered regarding childhood sexual abuse, and patients may have suffered another type of stressors.

In conclusion, patients hospitalized for SB includes those with a high risk of short-term relapse, and they are characterized by younger age, a history of previous SA, younger age at their first SA, and a higher frequency of the L-allele in the 5-HTTLPR. Therefore, this polymorphism can potentially be used as a predictive biomarker of this population.

\section{Funding}

Supported in part by a grant of the University of Buenos Aires (UBACYT 2013-2016: 20020120300022BA code Exp-UBA17, 064/ 2012) and by a grant from Ministry of Science, Technology and Productive Innovation of Argentina (PIDC-2012-0064).

\section{Disclosures}

No author or immediate family member has financial relationships with commercial entities that might appear to represent a potential for conflicts of interest. All authors have approved the final article.

\section{Acknowledgments}

The authors want to gratefully acknowledge the study participants for their collaboration, the nursing staff, and field teams in each hospital. Authors also want to thank Pamela Chan, $\mathrm{PhD}$, (Senior Medical Writer, Infusion Communications) for editorial assistance with the manuscript.

\section{References}

[1] World Health Organization. Suicide rates, age-standardized. 2015.

[2] Bostwick JM, Pabbati C, Geske JR, McKean AJ. suicide attempt as a risk factor for completed suicide: even more lethal than we knew. Am J Psychiatry 2016;173:1094-100.

[3] Finkelstein Y, Macdonald EM, Hollands S, Sivilotti ML, Hutson JR, Mamdani MM, et al. Risk of suicide following deliberate self-poisoning. JAMA Psychiatry 2015;72:570-5.

[4] Hawton K, van Heeringen K. Suicide. Lancet 2009;373:1372-81.

[5] Mann JJ, Waternaux C, Haas GL, Malone KM. Toward a clinical model of suicidal behavior in psychiatric patients. Am J Psychiatry 1999;156:181-9.

[6] Roy A, Rylander G, Sarchiapone M. Genetics of suicides. Family studies and molecular genetics. Ann N Y Acad Sci 1997:836:135-57.

[7] Voracek M, Loibl LM. Genetics of suicide: a systematic review of twin studies. Wien Klin Wochenschr 2007;119:463-75.

[8] Brent DA, Oquendo M, Birmaher B, Greenhill L, Kolko D, Stanley B, et al. Familial transmission of mood disorders: convergence and divergence with transmission of suicidal behavior. J Am Acad Child Adolesc Psychiatry 2004;43:1259-66.

[9] Kim CD, Seguin M, Therrien N, Riopel G, Chawky N, Lesage AD, et al. Familial aggregation of suicidal behavior: a family study of male suicide completers from the general population. Am J Psychiatry 2005;162:1017-9. 
[10] Sokolowski M, Wasserman J, Wasserman D. Genome-wide association studies of suicidal behaviors: a review. Eur Neuropsychopharmacol 2014;24:1567-77.

[11] Lutz PE, Mechawar N, Turecki G. Neuropathology of suicide: recent findings and future directions. Mol Psychiatry 2017;22:1395-412.

[12] Lockwood LE, Su S, Youssef NA. The role of epigenetics in depression and suicide: a platform for gene-environment interactions. Psychiatry Res 2015;228:235-42.

[13] Asberg M, Traskman L, Thoren P. 5-HIAA in the cerebrospinal fluid. A biochemical suicide predictor? Arch Gen Psychiatry 1976;33:1193-7.

[14] Mann JJ. The serotonergic system in mood disorders and suicidal behaviour. Philos Trans R Soc Lond B Biol Sci 2013;368:20120537.

[15] Mann JJ, Brent DA, Arango V. The neurobiology and genetics of suicide and attempted suicide: a focus on the serotonergic system. Neuropsychopharmacology 2001;24:467-77.

[16] Mirkovic B, Laurent C, Podlipski MA, Frebourg T, Cohen D, Gerardin P. Genetic association studies of suicidal behavior: a review of the past 10 years, progress, limitations, and future directions. Front Psychiatry 2016;7:158.

[17] Lesch KP, Wolozin BL, Estler HC, Murphy DL, Riederer P. Isolation of a cDNA encoding the human brain serotonin transporter. J Neural Transm Gen Sect 1993;91:67-72.

[18] Horstmann S, Binder EB. Pharmacogenomics of antidepressant drugs. Pharmacol Ther 2009;124:57-73.

[19] Gelernter J, Cubells JF, Kidd JR, Pakstis AJ, Kidd KK. Population studies of polymorphisms of the serotonin transporter protein gene. Am J Med Genet 1999;88:61-6.

[20] Heils A, Teufel A, Petri S, Stober G, Riederer P, Bengel D, et al. Allelic variation of human serotonin transporter gene expression. J Neurochem 1996;66:2621-4.

[21] Parsey RV, Hastings RS, Oquendo MA, Hu X, Goldman D, Huang YY, et al. Effect of a triallelic functional polymorphism of the serotonin-transporter-linked promoter region on expression of serotonin transporter in the human brain. Am J Psychiatry 2006;163:48-51.

[22] Courtet P, Picot MC, Bellivier F, Torres S, Jollant F, Michelon C, et al. Serotonin transporter gene may be involved in short-term risk of subsequent suicide attempts. Biol Psychiatry 2004;55:46-51.

[23] Mann JJ, Arango VA, Avenevoli S, Brent DA, Champagne FA, Clayton P, et al. Candidate endophenotypes for genetic studies of suicidal behavior. Biol Psychiatry 2009;65:556-63.

[24] Coccaro EF, Fanning JR, Phan KL, Lee R. Serotonin and impulsive aggression. CNS Spectr 2015:20:295-302.

[25] Davidge KM, Atkinson L, Douglas L, Lee V, Shapiro S, Kennedy JL, et al. Association of the serotonin transporter and 5HT1Dbeta receptor genes with extreme, persistent and pervasive aggressive behaviour in children. Psychiatr Genet 2004;14:143-6.

[26] Beitchman JH, Baldassarra L, Mik H, De Luca V, King N, Bender D, et al. Serotonin transporter polymorphisms and persistent, pervasive childhood aggression. Am J Psychiatry 2006;163:1103-5.

[27] Gerra G, Garofano L, Castaldini L, Rovetto F, Zaimovic A, Moi G, et al. Serotonin transporter promoter polymorphism genotype is associated with temperament, personality traits and illegal drugs use among adolescents. J Neural Transm (Vienna, Austria: 1996) 2005;112:1397-410.

[28] Cadoret RJ, Langbehn D, Caspers K, Troughton EP, Yucuis R, Sandhu HK, et al. Associations of the serotonin transporter promoter polymorphism with aggressivity, attention deficit, and conduct disorder in an adoptee population. Compr Psychiatry 2003;44:88-101.

[29] Sysoeva OV, Maluchenko NV, Timofeeva MA, Portnova GV, Kulikova MA, Tonevitsky AG, et al. Aggression and 5HTT polymorphism in females: study of synchronized swimming and control groups. Int J Psychophysiol 2009;72:173-8.

[30] Patkar AA, Berrettini WH, Hoehe M, Thornton CC, Gottheil E, Hill K, et al. Serotonin transporter polymorphisms and measures of impulsivity, aggression, and sensation seeking among African-American cocainedependent individuals. Psychiatry Res 2002;110:103-15.

[31] Silva H, Iturra P, Solari A, Villarroel J, Jerez S, Jimenez M, et al. Fluoxetine response in impulsive-aggressive behavior and serotonin transporter polymorphism in personality disorder. Psychiatr Genet 2010;20:25-30.

[32] Nock MK. The Oxford handbook of suicide and self-injury. Oxford University Press; 2014.

[33] Silverman MM, Berman AL, Sanddal ND, O'Carroll PW, Joiner TE. Rebuilding the tower of babel: a revised nomenclature for the study of suicide and suicidal behaviors. Part 2: suicide-related ideations, communications, and behaviors. Suicide Life Behav 2007;37:264-77.

[34] Ferrando L, Bobes J, Gibert JMINI. Mini International neuropsychiatric interview version en español 5.0.0. Sheehan DV \& lecrubier Y. 2004.

[35] Villar Garcia M, Perez Prieto JF, Hernandez Viadel M, Renovell Farre M, Leal Cercos C, Gomez Beneyto M. Preparation of a SCID-II-based diagnostic tool for personality disorders. Spanish version. Translation and adaptation. Actas Luso Neurol Psiquiatr Cienc Afines 1995;23:178-83.

[36] Posner K, Brown GK, Stanley B, Brent DA, Yershova KV, Oquendo MA, et al. The Columbia-suicide severity rating scale: initial validity and internal consistency findings from three multisite studies with adolescents and adults. Am J Psychiatry 2011;168:1266-77.

[37] Lopez PL, Cetkovich-Bakmas M, Lischinsky A, Alvarez Prado D, Torrente F. Psychometric properties of the barratt impulsiveness scale in a sample of the city of Buenos Aires. Vertex 2012;23:85-91.

[38] Oquendo MA, Graver R, Baca-García E, Morales M, Montalbán V, Mann JJ. Spanish adaptation of buss-durkee hostility inventory (BDHI). Eur J Psychiatry $2001 ; 15$.
[39] Mikulic IM, Cassullo GL, Crespi MC, Marconi A. Beck hopelessness scale BHS (A. Beck, 1974): psychometric study and standardization of Argentinian adaptation. nuario de investigaciones 2009;16.

[40] Brugha TS, Cragg D. The list of threatening experiences: the reliability and validity of a brief life events questionnaire. Acta Psychiatr Scand 1990;82:77-81.

[41] Roy A, Rylander G, Sarchiapone M. Genetic studies of suicidal behavior. Psychiatr Clin North Am 1997;20:595-611.

[42] Mann JJ, Malone KM. Cerebrospinal fluid amines and higher-lethality suicide attempts in depressed inpatients. Biol Psychiatry 1997;41:162-71.

[43] Sullivan GM, Ogden RT, Oquendo MA, Kumar JS, Simpson N, Huang YY, et al. Positron emission tomography quantification of serotonin-1A receptor binding in medication-free bipolar depression. Biol Psychiatry 2009;66:223-30.

[44] Oquendo MA, Galfalvy H, Sullivan GM, Miller JM, Milak MM, Sublette ME, et al. Positron emission tomographic imaging of the serotonergic system and prediction of risk and lethality of future suicidal behavior. JAMA Psychiatry 2016;73:1048-55.

[45] Lesch KP, Bengel D, Heils A, Sabol SZ, Greenberg BD, Petri S, et al. Association of anxiety-related traits with a polymorphism in the serotonin transporter gene regulatory region. Science (New York, NY) 1996;274:1527-31.

[46] Du L, Faludi G, Palkovits M, Demeter E, Bakish D, Lapierre YD, et al. Frequency of long allele in serotonin transporter gene is increased in depressed suicide victims. Biol Psychiatry 1999;46:196-201.

[47] Bellivier F, Szoke A, Henry C, Lacoste J, Bottos C, Nosten-Bertrand M, et al. Possible association between serotonin transporter gene polymorphism and violent suicidal behavior in mood disorders. Biol Psychiatry 2000; 48:319-22.

[48] Bondy B, Erfurth A, de Jonge S, Kruger M, Meyer H. Possible association of the short allele of the serotonin transporter promoter gene polymorphism (5-HTTLPR) with violent suicide. Mol Psychiatry 2000;5:193-5.

[49] Campi-Azevedo AC, Boson W, De Marco L, Romano-Silva MA, Correa H. Association of the serotonin transporter promoter polymorphism with suicidal behavior. Mol Psychiatry 2003:8:899-900.

[50] Courtet P, Baud P, Abbar M, Boulenger JP, Castelnau D, Mouthon D, et al. Association between violent suicidal behavior and the low activity allele of the serotonin transporter gene. Mol Psychiatry 2001;6:338-41.

[51] Gorwood P, Batel P, Ades J, Hamon M, Boni C. Serotonin transporter gene polymorphisms, alcoholism, and suicidal behavior. Biol Psychiatry 2000;48:259-64.

[52] Neves FS, Silveira G, Romano-Silva MA, Malloy-Diniz L, Ferreira AA, De Marco L, et al. Is the 5-HTTLPR polymorphism associated with bipolar disorder or with suicidal behavior of bipolar disorder patients? Am J Med Genet Part B Neuropsychiatr Genet 2008;147B:114-6.

[53] Preuss UW, Koller G, Soyka M, Bondy B. Association between suicide attempts and 5-HTTLPR-S-allele in alcohol-dependent and control subjects: further evidence from a German alcohol-dependent inpatient sample. Biol Psychiatry 2001:50:636-9.

[54] Wasserman D, Geijer T, Sokolowski M, Frisch A, Michaelovsky E, Weizman A, et al. Association of the serotonin transporter promotor polymorphism with suicide attempters with a high medical damage. Eur Neuropsychopharmacol 2007:17:230-3.

[55] Courtet P, Buresi C, Abbar M, Baud P, Boulenger JP, Castelnau D, et al. No association between non-violent suicidal behavior and the serotonin transporter promoter polymorphism. Am J Med Genet Part B Neuropsychiatr 2003;116B:72-6.

[56] Chong SA, Lee WL, Tan CH, Tay AH, Chan AO, Tan EC. Attempted suicide and polymorphism of the serotonin transporter gene in Chinese patients with schizophrenia. Psychiatry Res 2000;97:101-6.

[57] Fitch D, Lesage A, Seguin M, Trousignant M, Bankelfat C, Rouleau GA, et al. Suicide and the serotonin transporter gene. Mol Psychiatry 2001;6:127-8.

[58] Mann JJ, Huang YY, Underwood MD, Kassir SA, Oppenheim S, Kelly TM, et al. A serotonin transporter gene promoter polymorphism (5-HTTLPR) and prefrontal cortical binding in major depression and suicide. Arch Gen Psychiatry 2000;57:729-38.

[59] Iurescia S, Seripa D, Rinaldi M. Looking beyond the 5-HTTLPR polymorphism: genetic and epigenetic layers of regulation affecting the serotonin transporter gene expression. Mol Neurobiol 2017;54:8386-403.

[60] Schneider I., Kugel H. Association of Serotonin Transporter Gene AluJb Methylation with major Depression, Amygdala Responsiveness, 5-HTTLPR/ rs25531 Polymorphism, and Stress. 2018;43:1308-1316.

[61] Greenberg BD, Tolliver TJ, Huang SJ, Li Q, Bengel D, Murphy DL. Genetic variation in the serotonin transporter promoter region affects serotonin uptake in human blood platelets. Am J Med Genet 1999;88:83-7.

[62] Gaspar P, Cases O, Maroteaux L. The developmental role of serotonin: news from mouse molecular genetics. Nat Rev Neurosci 2003;4:1002-12.

[63] Gould E. Serotonin and hippocampal neurogenesis. Neuropsychopharmacology 1999;21:46S-51S.

[64] Jedema HP, Gianaros PJ, Greer PJ, Kerr DD, Liu S, Higley JD, et al. Cognitive impact of genetic variation of the serotonin transporter in primates is associated with differences in brain morphology rather than serotonin neurotransmission. Mol Psychiatry 2010;15:512-22 446.

[65] Duman RS. Pathophysiology of depression: the concept of synaptic plasticity. Eur Psychiatry 2002;17(Suppl 3):306-10.

[66] Frodl T, Koutsouleris N, Bottlender R, Born C, Jager M, Morgenthaler M, et al, Reduced gray matter brain volumes are associated with variants of the serotonin transporter gene in major depression. Mol Psychiatry 2008;13:1093-101. 
[67] Wiggins JL, Bedoyan JK, Peltier SJ, Ashinoff S, Carrasco M, Weng SJ, et al. The impact of serotonin transporter (5-HTTLPR) genotype on the development of resting-state functional connectivity in children and adolescents: a preliminary report. Neuroimage 2012;59:2760-70.

[68] Shinozaki G, Romanowicz M, Passov V, Rundell J, Mrazek D, Kung S. State dependent gene-environment interaction: serotonin transporter gene-child abuse interaction associated with suicide attempt history among depressed psychiatric inpatients. J Affect Disord 2013;147:373-8.

[69] Wagner S, Baskaya O, Lieb K, Dahmen N, Tadic A. The 5-HTTLPR polymorphism modulates the association of serious life events (SLE) and impulsivity in patients with borderline personality disorder. J Psychiatr Res 2009;43: 1067-72.

[70] Hu XZ, Lipsky RH, Zhu G, Akhtar LA, Taubman J, Greenberg BD, et al. Serotonin transporter promoter gain-of-function genotypes are linked to obsessivecompulsive disorder. Am J Hum Genet 2006;78:815-26.

[71] Haberstick BC, Smolen A, Williams RB, Bishop GD, Foshee VA, Thornberry TP, et al. Population frequencies of the triallelic 5HTTLPR in six ethnicially diverse samples from North America, Southeast Asia, and Africa. Behav Genet 2015;45:255-61. 